\title{
Fatigue failure load of monolithic Y-TZP three-unit-fixed dental prostheses: Effect of grinding at the gingival zone of the connector
}

\author{
Marina Amaral $^{\mathrm{a}}$, Regina F. Villefort ${ }^{\mathrm{b}}$, Renata Marques Melo ${ }^{\mathrm{b}}$, Gabriel K.R. Pereira ${ }^{\mathrm{c}}$, Yu Zhang ${ }^{\mathrm{d}}$, \\ Luiz Felipe Valandro ${ }^{\mathrm{c}, *}$, Marco Antonio Bottino ${ }^{\mathrm{b}}$ \\ a Department of Dentistry, Dental Prosthesis, University of Taubate, Taubaté, São Paulo State, Brazil \\ b Post Graduation Program in Restorative Dentistry (Prosthetic Dentistry Unit), School of Dentistry, Sao Paulo State University, São José dos Campos, São \\ Paulo State, Brazil \\ ${ }^{c}$ Post Graduation Program in Dental Sciences (Prosthetic Dentistry Unit), School of Dentistry, Federal University of Santa Maria, Santa Maria, Rio Grande \\ do Sul State, Brazil \\ d Department of Biomaterials and Biomimetics, New York University College of Dentistry, 433 First Avenue, New York, NY, USA
}

\section{A R T I C L E I N F O}

\section{Keywords:}

Fixed prosthodontics

Zirconium oxide partially stabilized by yttrium Grinding

Fatigue

\begin{abstract}
A B S T R A C T
Objectives: To determine the fatigue failure loads of three-unit monolithic zirconia fixed dental prosthesis (FDPs) before and after grinding of the gingival areas of connectors with diamond burs.

Material and methods: FDPs were milled from pre-sintered blocks of zirconia simulating the absence of the first mandibular molar. Half of the specimens were subjected to grinding, simulating clinical adjustment, and all of them were subjected to glazing procedure. Additional specimens were manufactured for roughness analysis. FDPs were adhesively cemented onto glass-fiber reinforced epoxy resin abutments. Fatigue failure loads and standard deviations were obtained using a staircase fatigue method $(n=20,100,000$ loading cycles $/ 5 \mathrm{~Hz})$. The initial test load was $70 \%$ of the mean load-to-fracture $(n=3)$ and load increments were $5 \%$ of the initial test load for both the control and ground specimens. Data were compared by Student's $T$-test $(\alpha \leq 0.05)$.

Results: Both the control and ground groups exhibited similar values of load-to-fracture and fatigue failure loads. Neither the surface treatments nor ageing affected the surface roughness of the specimens.

Conclusions: The damage induced by grinding with fine-grit diamond bur in the gingival area of the connectors did not decrease the fatigue failure load of the three-unit monolithic zirconia FDP.
\end{abstract}

\section{Introduction}

The report of fracture at the connector of lithium disilicate fixed dental prosthesis (FDP) are common in literature (Solá-Ruiz et al., 2013; Makarouna et al. 2011., Wolfart et al., 2009). The need of a more resistant material for fabrication all-ceramic FDP makes zirconia-based ceramics the material of choice, due to their excellent mechanical, esthetic, and biological characteristics (Lee et al., 2011; Della Bona and Kelly, 2008). Since zirconia is relatively opaque, the use of a glassceramic overlay was still necessary to enhance esthetics (Della Bona and Kelly, 2008). However, the main reason for clinical failure of such restorations is chipping and delamination of porcelain veneers (Sailer et al., 2009; Schmitt et al.,2012). And major chipping was observed before fracture of framework (Salido et al., 2012). Much effort has been devoted to the development of zirconia blocks with enhanced esthetics in order to completely eliminate the veneering step and mill fullcontour (monolithic) restorations (Johansson et al., 2014).
After milling and before the cementation of FDPs, clinical adjustments are commonly required to obtain an appropriate occlusal relation and gingival profile (Aboushelib et al., 2009). Damages, as scratches on the zirconia surface, caused by grinding or even polishing procedures may promote the crystalline phase transformation from tetragonal to monoclinic phase (Kim et al., 2010). The relation between the size of the resulting scratches and cracks and the depth of the transformed layer characterizes the crack growth and determines whether the material will fail or not at loads below the critical load value (Kim et al., 2010).

For measurement of zirconia mechanical properties after surface alterations, most available literature uses geometric planar specimens (i.e. discs). For $1.2 \mathrm{~mm}$ Y-TZP discs, the abrasion of the ceramic surface with smaller grit sizes did not affected the biaxial flexural strength after ageing (Pereira et al., 2016a). However, this specimen configuration does not consider the curved surface of the connector in a FDP, or the variations in material's thickness along the crowns, not

\footnotetext{
* Correspondence to: Federal University of Santa Maria, Faculty of Odontology, R. Floriano Peixoto, 1184, 97015-372 Santa Maria, Rio Grande do Sul State, Brazil.

E-mail address: lfvalandro@hotmail.com (L.F. Valandro).
} 
reflecting the stress distribution present in clinical situations (Hooi et al., 2014).

The second issue that affects monolithic zirconia structures is lowtemperature degradation (LTD) in humid environments, which is also related to the crystalline transformation from the tetragonal to monoclinic phase (Pereira et al., 2016a). As a result of LTD, it was observed grain dislodgements (resulting from the volume expansion associated with the phase transformation), increase on surface roughness (Yoshimura et al., 1987) and decrease of strength, toughness, and density of the material (Pereira et al., 2015; Kim et al., 2009).

Therefore, the purpose of this study was to evaluate the fatigue failure loads of three-unit FDP with or without grinding of the connector gingival areas by fine grit-size diamond burs. The null hypothesis is that grinding do not affect the fatigue failure load of zirconia FDP.

\section{Material and methods}

\subsection{Production of monolithic Y-TZP 3-unit-fixed dental prostheses}

Pre-sintered blocks containing 3 mol\% of zirconia (VITA In Ceram YZ, Vita Zahnfabrik) were used to mill 46 three-unit FDPs, simulating the absence of the first mandibular molar with abutments on the second mandibular premolar and second mandibular molar.

The abutments with simplified geometries were milled from glassfiber reinforced epoxy resin (NEMA grade G10, Accurate Plastics Inc.) and had heights of $6 \mathrm{~mm}$, circumferential chamfers of $120^{\circ}$ (radius $=0.5 \mathrm{~mm}$ ), and total occlusal convergence angles of $6^{\circ}$. All transitions from the axial to the occlusal surfaces were rounded, and central mesial-distal fissures were simulated on the occlusal surface. The abutments were embedded into polyurethane (F 16 e F 16 ISO, Axson Technologies) at distances of $17 \mathrm{~mm}$ from each other.

The obtained sets were scanned (InEos, Sirona), and the corresponding three-unit FDPs were drawn in a CAD system (inLab SW4.2, Sirona). The minimum wall thicknesses were of: $0.7 \mathrm{~mm}$ occlusal (central fossa region), $1.5 \mathrm{~mm}$ in cusps, 0.5 circumferential, $0.2 \mathrm{~mm}$ marginal, and connectors (area between crowns) with mean sectional area of 22,5 $\mathrm{mm}^{2}$. The restorations were then milled (CEREC InLab MC XL instrument, Sirona).

Restorations were sintered according to manufacturer instructions $\left(1530^{\circ} \mathrm{C} / 120 \mathrm{~min}\right)$ in a Vita Zyrcomat $\mathrm{T}$ furnace (Vita Zahnfabrik) and subjected to the following subsequent treatments: control group $(\mathrm{n}=23)$ (Villefort et al., 2017): the specimens were coated with a glaze layer (sintering temperature/time: $900{ }^{\circ} \mathrm{C} / 60 \mathrm{~s}$ ) on the external surface (VITA Akzent Plus Glaze, Vita Zahnfabrik); grinding group $(\mathrm{n}=23)$ : firstly the bridges were ground by a single trained operator under water cooling with diamond burs $(30 \mu \mathrm{m}$, 3215FF, KG Sorensen): the diamond bur was positioned at the gingival area of the connectors and movements were performed on the buccal-lingual way, respecting the anatomy of the area, until all surface was completely treated by the bur. Cautious was taken to not reduce the connectors sectional area. A new diamond bur was used for each specimen. After, the specimens were coated with the glaze layer, as described for control group.

\subsection{Cementation}

The epoxy-resin abutments were treated with hydrofluoric acid (10\% Condac Porcelana, FGM) for $1 \mathrm{~min}$, rinsed, dried, and then coated with silane (RelyX ${ }^{\mathrm{TM}}$ Ceramic Primer, 3M ESPE). The intaglio surfaces of the restorations were first abraded with $30-\mu \mathrm{m}$ silicamodified alumina particles (Rocatec Soft, 3M ESPE) and then coated with silane. Dual-cure resin cement was applied to the abutments (Variolink II, Ivoclar Vivadent AG), and the FDPs were placed under a load of $750 \mathrm{~g}$. The excess cement was removed with a brush, and the assembly was light-activated (Radii-Cal, SDI) for $40 \mathrm{~s}$ on the buccal, lingual, and occlusal surfaces of each abutment crown. After, the bottom part of abutments was embedded into polyurethane ( $\mathrm{F}$ 16Polyol and F 16 ISO, Axson Technologies).

\subsection{Fatigue failure load testing - staircase method}

Three specimens from each group were initially fractured under monotonic loading (at a speed of $1 \mathrm{~mm} / \mathrm{min}$ ) using a universal testing machine (Emic DL 1000, Emic) to determine load-to-fracture. The specimens were positioned at $30^{\circ}$ angles with respect to the long axis and immersed into water. The load was applied to the inner inclines of the buccal cusps through a tungsten carbide round tip (with diameter of $6 \mathrm{~mm}$ ) until fracture.

Staircase tests $(n=20)$ were performed in a dynamic electric machine (Instron Electropuls E3000, Instron): the initial load was defined as $70 \%$ of the mean load-to-fracture for each group. The load increments (step size) were equal to $5 \%$ of the initial load, and 100,000 testing cycles were performed at a frequency of $5 \mathrm{~Hz}$. The first specimen was tested at the initial load; if it was fractured after 100,000 cycles, the next specimen was tested at the initial load decreased by one load increment. However, if the first specimen endured, the next specimen was tested at the initial load increased by one load increment. Such a design produces a graphical appearance of a stair represented by up-and-down lines. Student $T$-test was performed to compare the fatigue failure load of both groups $(\alpha \leq 0.05)$.

\subsection{Fractographic analysis}

Failure analyses of the fractured specimens were conducted for specimens tested in both load-to-fracture and staircase testing. The fracture origins were identified and classified according to their locations in the FDP. The related analyses were performed by the stereomicroscope (Discovery V20, Carl-Zeiss).

\subsection{Roughness measurement}

Additional specimens $(n=5)$ were fabricated from control and grinding groups for measurement of surface roughness. After sectioning of the blocks of zirconia (VITA In Ceram YZ, Vita Zahnfabrik) into plates $(0.5 \mathrm{~mm} \times 1.0 \mathrm{~mm} \times 1.0 \mathrm{~mm}$ thickness $)$, surface was finished with polishing (1200 grit) in green stage and sintered (Vita Zyrcomat $\mathrm{T}$ furnace, Vita Zahnfabrik; $1530{ }^{\circ} \mathrm{C} / 120 \mathrm{~min}$ ). Specimens from grinding group received abrasion with diamond burs, as mentioned previously. Roughness was calculated by the mean of 3 parallel readings, and parameters $\mathrm{Ra}$ (average roughness) and $\mathrm{Rz}$ (mean roughness depth) were reported. Student $T$-test $(\alpha \leq 0.05)$ was used for comparison of parameters between groups.

\section{Results and discussion}

\subsection{Fatigue failure load: staircase testing}

The fracture loads $(n=3)$ obtained for the two tested groups were used for determination of staircase parameters (Table 1). The null hypothesis was accepted: groups exhibited similar fatigue failure load values according to Student's $t$-test $(\mathrm{p}=0.126)$. Damage group presented a greater decrease from the load-to-fracture to the mean fatigue failure load (18.0\%) than control group (14.6\%) (Table 1).

Damage of zirconia surface after sintering may decrease (Kim et al., 2009) or not (Pereira et al., 2015; Amaral et al., 2015) the strength of the pieces. Preliminary damage of zirconia frameworks does not have a detrimental effect on the load-bearing capacity of the specimens (Kohorst et al., 2010). The same for silicatization of zirconia, as surface treatment, of geometrical specimens (Amaral et al., 2015) or copings (Campos et al., In press). 
Table 1

Load-to-fracture (in Newton), initial load to start the fatigue test (in Newton) and load increment (in Newton) for staircase test. Mean fatigue failure load and respective standard deviations and the decrease of mean fatigue failure load from load-to-fracture.

\begin{tabular}{llllll}
\hline & $\begin{array}{l}\text { Fracture } \\
\text { load (N) }\end{array}$ & $\begin{array}{l}\text { Initial } \\
\text { load } \\
\text { (N) }\end{array}$ & $\begin{array}{l}\text { Load } \\
\text { increment } \\
\text { (N) }\end{array}$ & $\begin{array}{l}\text { fatigue } \\
\text { failure } \\
\text { loads } \\
\text { (N) }\end{array}$ & $\begin{array}{l}\text { Decrease } \\
\text { from the } \\
\text { load to } \\
\text { fracture } \\
\text { (\%) }\end{array}$ \\
\hline Control & 1907.66 & 1335.36 & 66.77 & $\begin{array}{l}1629.14 \\
(152.25)\end{array}$ & 14.6 \\
Grinding & 2069.37 & 1448.56 & 72.43 & $\begin{array}{l}1696.88 \\
(124.55)\end{array}$ & 18.0 \\
\hline
\end{tabular}

The geometrical complexity of prosthetic restorations, as compared to specimens of in vitro studies (bars and discs), may influence the stress field generated and result in different behavior of the material (Hooi et al., 2014). This is the reason why this study selected the smoothest abrasion procedure (extra-fine diamond burs) to damage FDP. Fatigue stimuli may promote crack propagation into the bulk of the material. Hence, in addition to the results presented in this study, the literature (Amaral et al., 2015) data also demonstrated that zirconia restorations exhibited high tolerance to mechanical damages. Grinding conditions may affect the fatigue behavior of the abraded ceramics (such as grinding pressure, speed and grit size of the grinding tool, as also the presence/absence of cooling) (Pereira et al., 2016b). The more harmful the protocol (high speed, large grit sizes, or absence of proper cooling) higher the risk of a deleterious impacts on the mechanical properties of the zirconia ceramics (Kim et al. 2009). The protocol employed in this study was the recommended by the literature (Pereira et al., 2016a) for clinical adjustment of Y-TZP pieces.

However, these are not the only factors affecting zirconia mechanical properties: surface topography and roughness should also be taken into account. In this study, abrasion was followed by the application of a glaze layer; thus, the exposed surface was similar to those of the control specimens, producing similar results for both groups.

\subsection{Fractographic analysis}

The results of the failure analysis revealed the existence of three main regions of failure origins for both groups: the buccal area of the connector between the two molars, the gingival area of the connector, and the area of load application, which was in contact with the tungsten carbide tip (Fig. 1). The main failure was observed in the buccal area of the connector (two specimens from each group in the load-to-fracture test, and three specimens from each group in the staircase test, Fig. 1A). Failures at the occlusal surface of the pontic (load application site, Fig. 1B) occurred only during staircase test (1 specimen per group). Failures in the gingival area of the connector (Fig. 1C) were observed during both load-to-fracture (one specimen per group) and fatigue testing (control: 2 specimens; damage 1 specimen).

Failure at connector areas (buccal or gingival) was found to be mainly at the distal connector between the pontics (connector between molars) (Fig. 1A, C and D). A previous finite element analysis revealed that this same area presents the highest stress concentration on a three-unit lithium disilicate FDP, where the moment arm is longer (Oh et al., 2002). The connector is the main fracture site of FDPs (Makarouna et al., 2011; Solá-Ruiz et al., 2013; Schmitt et al., 2012). Very similar failure origin (buccal side of the distal connector - lithium disilicate FDP) was obtained clinically and reported by Wolfart et al. (2009).

The failures seen during load-to-fracture and staircase fatigue testing were always catastrophic, producing three or more fragments as a result of the high loads applied (Oh et al., 2002). Clinically, these types of failure would result in the removal and replacement of the restorations. Only one specimen from each group failed from the occlusal surface (load application point). No loss of retention during fatigue was recorded, despite the challenges presented by zirconia for bonding (clinical annual complication rates of $1.28 \%$ and 5 -year complication rates of 6.2\%) (Pjetursson et al., 2015).

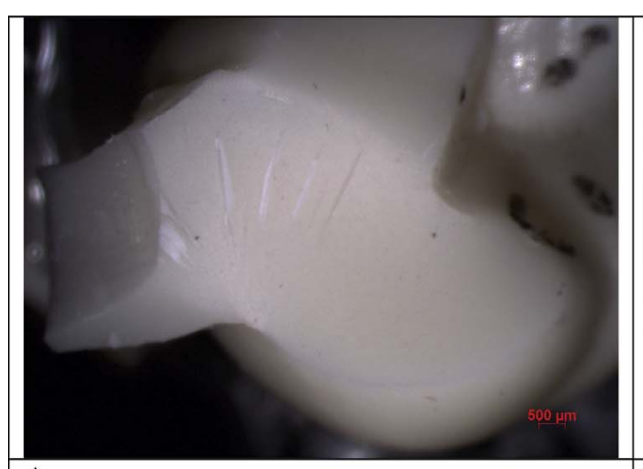

A

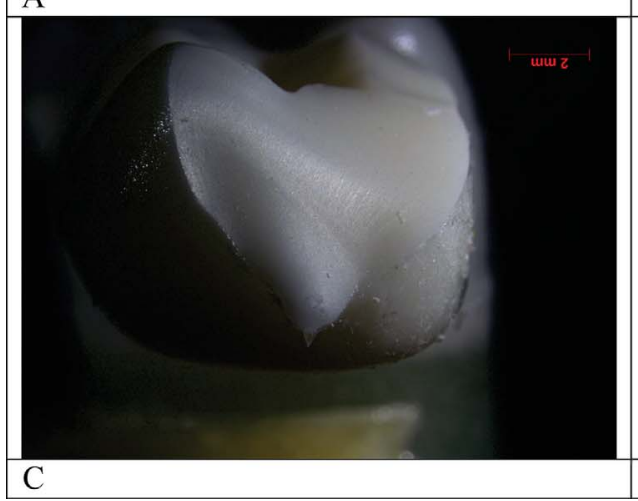

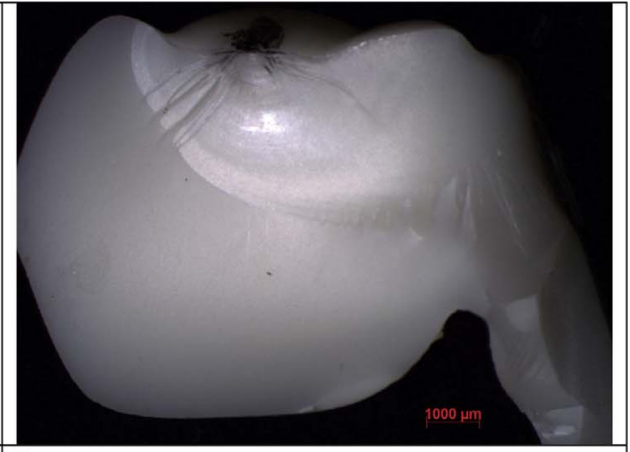

B

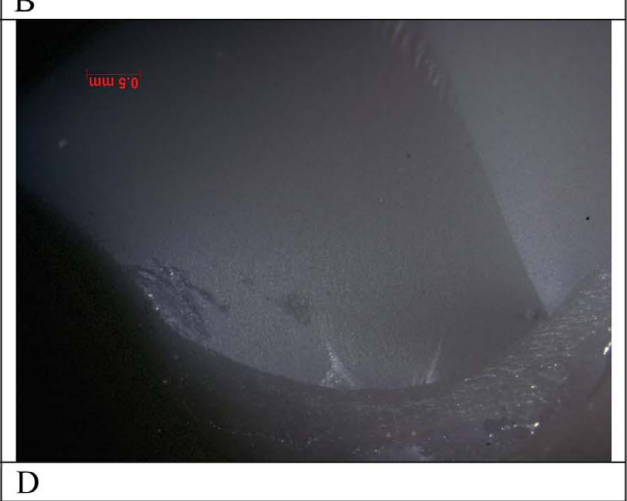

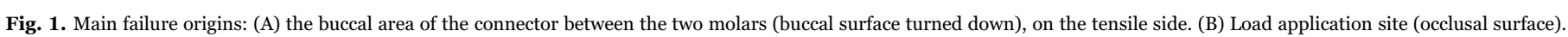
(C) The gingival area of the connector (tensile side). (D) Detailed failure origin of (C) at the gingival area of the connector. 


\subsection{Roughness analysis}

The small damage caused by grinding in the present study was confirmed by the roughness results. The roughness caused by extra-fine diamond bur $(30 \mu \mathrm{m})$ and the irregularities from glaze layer produced similar Ra and $\mathrm{Rz}$ values, which may have collaborated for the similar results of fatigue failure load between groups. Both $\mathrm{Ra}$ and $\mathrm{Rz}$ parameters were similar between control (Ra: $1.13 \mu \mathrm{m} \pm 0.1$; Rz: $9.27 \mu \mathrm{m} \pm 4.8$ ) and grinding (Ra: $0.80 \mu \mathrm{m} \pm 0.3$; Rz: $7.54 \mu \mathrm{m} \pm 4.1$ ) groups (p-value $\mathrm{Ra}=0.057 ; \mathrm{Rz}=0.558$ ).

Zirconia is a suitable material for fabricating frameworks and monolithic restorations, bearing high loads even under fatigue (Table 1). Adjustments of monolithic Y-TZP restorations after sintering, when performed according to the tested protocol, do not decrease the fatigue failure load of the restoration. But, it highlights that the dimensions recommended by manufacturer for milling ceramic structures must always be followed. The simplified abutment design may be a drawback of this study, especially when the marginal configuration is considered (as compared to the clinical situation).

\section{Conclusion}

The adjustment by grinding of the connector area of three-unit fullcontour zirconia FDP followed by glazing did not damage the fatigue failure load of the restorations. Thus, it might be safely performed, even in critical areas.

\section{Acknowledgement}

This work was supported by the National Counsel of Technological and Scientific Development [Grant number CNPQ/PDJ 150931/20140]; and the United States National Institutes of Health, National Institute of Dental and Craniofacial Research (Grant no. 2R01 DE017925).

\section{References}

Aboushelib, M.N., Feilzer, A.J., Kleverlaan, C.J., 2009. Bridging the gap between clinical failure and laboratory fracture strength tests using a fractographic approach. Dent. Mater. 25, 383-391.

Amaral, M., Cesar, P.F., Bottino, M.A., Lohbauer, U., Valandro, L.F., 2015. Fatigue behavior of Y-TZP ceramic after surface treatments. J. Mech. Behav. Biomed. Mater. 15, 149-156.

Campos, F., Valandro, L.F., Feitosa, S.A., Kleverlaan, C.J., Feilzer, A.J., de Jager, N., Bottino, M.A., 2017. Adhesive cementation promotes higher fatigue resistance to zirconia crowns. Oper. Dent. 42, 215-224, (In press).

Della Bona, A., Kelly, J.R., 2008. The clinical success of all-ceramic restorations. JADA
139, 8S-13S.

Hooi, P., Addison, O., Fleming, G.J.P., 2014. Strength determination of brittle materials as curved monolithic structures. J. Dent. Res. 93, 412-416.

Johansson, C., Kmet, G., Rivera, J., Larsson, C., Vult Von, Steyern, P., 2014. Fracture strength of monolithic all-ceramic crowns made of high translucent yttrium oxidestabilized zirconium dioxide compared to porcelain-veneered crowns and lithium disilicate crowns. Acta Odontol. Scand. 72, 145-153.

Kim, H.T., Han, J.S., Yang, J.H., Lee, J.B., Kim, S.H., 2009. The effect of low temperature aging on the mechanical property \& phase stability of Y-TZP ceramics. J. Adv. Prosthodont. 1, 113-117.

Kim, J.W., Covel, N.S., Guess, P.C., Rekow, E.D., Zhang, Y., 2010. Concerns of hydrothermal degradation in CAD/CAM zirconia. J. Dent. Res. 89, 91-95.

Kohorst, P., Butzheinen, L.O., Dittmer, M.P., Heuer, W., Borchers, L., Stiesch, M., 2010. Influence of preliminary damage on the load-bearing capacity of zirconia fixed dental prostheses. J. Prosthodont. 19, 606-613.

Lee, B.C., Jung, G.Y., Kim, D.J., Han, J.S., 2011. Initial bacterial adhesion on resin, titanium and zirconia in vitro. J. Adv. Prosthodont. 3, 81-84.

Makarouna, M., Ullmann, K., Lazarek, K., Boening, K.W., 2011. Six-year clinical performance of lithium disilicate fixed partial dentures. Int. J. Prosthodont. 24, 204-206.

Oh, W., Anusavice, K.J., 2002. Effect of connector design on the fracture resistance of allceramic fixed partial dentures. J. Prosthet. Dent. 87, 536-542.

Pereira, G.K., Silvestri, T., Amaral, M., Rippe, M.P., Kleverlaan, C.J., Valandro, L.F., 2016a. Fatigue limit of polycrystalline zirconium oxide ceramics: effect of grinding and low-temperature aging. J. Mech. Behav. Biomed. Mater. 19 (61), 45-54.

Pereira, G.K.R., Amaral, M., Cesar, P.F., Bottino, M.C., Kleverlaan, C.J., Valandro, L.F., 2015. Effect of low-temperature aging on the mechanical behavior of ground Y-TZP. J. Mech. Behav. Biomed. Mater. 45, 183-192.

Pereira, G.K.R., Fraga, S., Montagner, A.F., Soares, F.Z.M., Kleverlaan, C.J., Valandro, L.F., 2016b. The effect of grinding on the mechanical behavior of Y-TZP ceramics: a systematic review and meta-analyses. J. Mech. Behav. Biomed. Mater. 63, 417-442.

Pjetursson, B.E., Sailer, I., Makarov, N.A., Zwahlen, M., Thoma, D.S., 2015. All-ceramic or metal-ceramic tooth-supported fixed dental prostheses (FDPs)? A systematic review of the survival and complication rates. Part II: multiple-unit FDPs. Dent. Mater. 31, 624-639.

Sailer, I., Gottnerb, J., Kanelb, S., Hammerle, C.H., 2009. Randomized controlled clinical trial of zirconia-ceramic and metal-ceramic posterior fixed dental prostheses: a 3year follow-up. Int. J. Prosthodont. 22, 553-560.

Salido, M.P., Martinez-Rus, F., del Rio, F., Pradies, G., Ozcan, M., Suarez, M.J., 2012. Prospective clinical study of zirconia-based posterior four-unit fixed dental prostheses: four-year follow-up. Int. J. Prosthodont. 25, 403-409.

Schmitt, J., Goellner, M., Lohbauer, U., Wichmann, M., Reich, S., 2012. Zirconia posterior fixed partial dentures: 5-year clinical results of a prospective clinical trial. Int. J. Prosthodont. 25, 585-589.

Solá-Ruiz, M.F., Lagos-Flores, E., Román-Rodriguez, J.L., Highsmith Jdel, R., FonsFont, A., Granell-Ruiz, M., 2013. Survival rates of a lithium disilicate-based core ceramic for three-unit esthetic fixed partial dentures: a 10-year prospective study. Int. J. Prosthodont. 26, 175-180.

Villefort, R.F., Amaral, M., Pereira, G.K.R., Campos, T.M., Zhang, Y., Bottino, M.A. Valandro, L.F., de Melo, R.M., 2017. Effects of two grading techniques of zirconia material on the fatigue limit of full-contour 3-unit fixed dental prostheses. Dent. Mater 33 (4), e155-e164. http://dx.doi.org/10.1016/j.dental.2016.12.010.

Wolfart, S., Eschbach, S., Scherrer, S., Kern, M., 2009. Clinical outcome of three-unit lithium-disilicate glass-ceramic fixed dental prostheses: up to 8 years results. Dent. Mater. 25, e63-e71.

Yoshimura, M., Noma, T., Kawabata, K., Somiya, S., 1987. Role of H2O on the degradation process of Y-TZP. J. Mater. Sci. Lett. 6, 465-467. 